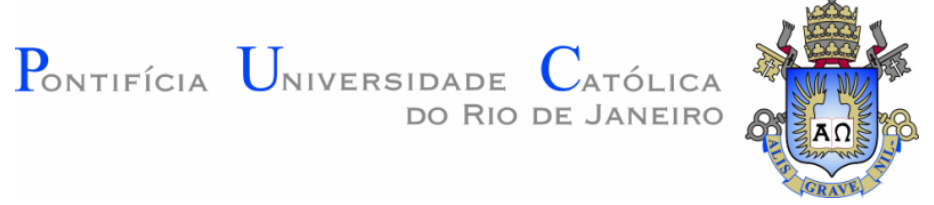

Juan José Cruz Villanueva

\begin{abstract}
Estudo Experimental da distribuição de fuligem e de hidrocarbonetos aromáticos policíclicos em chamas laminares não pré-misturadas de etileno e de ar
\end{abstract}

Tese de Doutorado

Tese apresentada ao Programa de Pós-graduação em Engenharia Mecânica da PUC-Rio como requisito parcial para obtenção do grau de Doutor em Engenharia Mecânica.

Orientador: Prof. Luís Fernando Figueira da Silva Co-orientador: Prof. Andrés Fuentes Castillo 
Juan José Cruz Villanueva

\section{Estudo Experimental da distribuição de fuligem e de hidrocarbonetos aromáticos policíclicos em chamas laminares não pré-misturadas de etileno e de ar}

Tese apresentada como requisito parcial para a obtenção do grau de Doutor pelo Programa de Pós-graduação em Engenharia Mecânica da PUC-Rio. Aprovada pela Comissão Examinadora abaixo assinada.

Prof. Luís Fernando Figueira da Silva Orientador Departamento de Engenharia Mecânica - PUC-Rio

Prof. Andrés Fuentes Castillo Co-Orientador Universidad Técnica Federico Santa Maria de Chile - USM

Prof. Amir Antônio Martins de Oliveira Júnior Universidade Federal de Santa Catarina - UFSC

Dr. Ricardo Serfaty Centro de Pesquisas - PETROBRAS

Dr. Paulo Roberto Pagot Centro de Pesquisas - PETROBRAS

Prof. Luís Fernando Alzuguir Azevedo Departamento de Engenharia Mecânica - PUC-Rio

Prof. Marcio da Silveira Carvalho Coordenador Setorial do Centro Técnico Científico - PUC-Rio 
Todos os direitos reservados. É proibida a reprodução total ou parcial do trabalho sem autorização da universidade, do autor e do orientador.

\section{Juan José Cruz Villanueva}

Graduou-se em Engenharia Mecânica na Universidade Nacional de Trujillo - Perú em 2009. Fez mestrado em Engenharia Mecânica na Pontifícia Universidade Católica do Rio de Janeiro - Brasil. Possui experiência profissional como Engenheiro de Mecânico na área de manutenção em diversas empresas peruanas, na área pesqueira e mineração.

Ficha Catalográfica

Cruz Villanueva, Juan José

Estudo experimental da distribuição de fuligem e de hidrocarbonetos aromáticos policíclicos em chamas laminares não pré-misturadas de etileno e de ar / Juan José Cruz Villanueva; orientador: Luís Fernando Figueira da Silva; co-orientador: Andrés Fuentes Castillo. - 2017.

141 f.: il. color.; $30 \mathrm{~cm}$

Tese (doutorado)-Pontifícia Universidade Católica do Rio de Janeiro, Departamento de Engenharia Mecânica, 2017.

Inclui bibliografia

1. Engenharia Mecânica - Teses. 2. Incandescência induzida por laser. 3. Fluorescência induzida por plano laser. 4. Absorção/emissão moduladas. 5. Atenuação na linha de visada. 6. Deconvolução. I. Silva, Luís Fernando Figueira da. II. Fuentes Castillo, Andrés. III. Pontifícia 
Dedicado às mulheres da minha vida: Sabina, Cecilia e Raphaella. 


\section{Agradecimentos}

À PUC-Rio, pela concessão de isenção de pagamento no programa de Doutorado em Engenharia Mecânica.

Ao $\mathrm{CNPq}$, pela concessão da bolsa de Doutorado e taxa de bancada para a realização deste estudo.

Ao Departamento de Engenharia Mecânica da PUC-Rio e ao Centro de Pesquisa da Petrobras, pela oportunidade de trabalhar no Projeto Conveniado "Desenvolvimento de uma modelagem da combustão em escoamento turbulento baseada em técnica euleriana/lagrangiana".

A meu orientador, Professor Luís Fernando Figueira da Silva pelo apoio, confiança, estímulo e dedicação para a realização deste trabalho.

A meu Co-orientador, Professor Andrés Fuentes Castillo pelos sugestões e aportes na realização deste trabalho.

Ao Dr. Ricardo Serfaty, obrigado pela confiança e auxílios, como responsável do Projeto Conveniado entre a PUC-Rio e a Petrobras, ao longo destes quatro anos.

Ao Professor Amir Oliveira, obrigado pelos empréstimos do queimador McKenna e dos medidores de vazão.

Ao meu colega e amigo Anthony Roque, pelas discussões, aportes e sugestões nas fases iniciais deste trabalho.

Aos meus colegas da PUC-Rio. 


\section{Resumo}

Cruz Villanueva, Juan José; Figueira da Silva, Luís Fernando (Orientador); Fuentes Castillo, Andrés (Co-orientador). Estudo experimental da distribuição de fuligem e de hidrocarbonetos aromáticos policíclicos em chamas laminares não pré-misturadas de etileno e de ar. Rio de Janeiro, 2017. 141p. Tese de Doutorado - Departamento de Engenharia Mecânica, Pontifícia Universidade Católica do Rio de Janeiro.

O presente trabalho apresenta um estudo experimental da distribuição da fuligem e de hidrocarbonetos aromáticos policíclicos (PAH) em chamas laminares não pré-misturadas de etileno e ar, mediante o uso de técnicas de diagnóstico espectroscópico, num queimador tipo co-flow. Para este fim são aplicadas as técnicas de fluorescência induzida por plano laser e incandescência induzida por plano laser, com excitação no espectro ultravioleta. Bandas espectrais de detecção centradas em 340, 400, 450, 500, $550 \mathrm{~nm}$ são empregadas para caracterizar diferentes PAH, aproveitado o fenômeno do deslocamento do espectros de fluorescência para o infravermelho, conforme se incrementa sua massa molecular. A técnica de extinção laser é utilizada para calibrar os resultados de incandescência e obter a fração volumétrica de fuligem. A radiação espontânea emitida pela fuligem é utilizada para medir a temperatura pela técnica de termometria em duas cores. A comparação dos resultados obtidos com uma detecção simultânea (0 ns) e atrasada (50 $n s)$ com respeito ao pulso laser permite discriminar entre as regiões onde estão presentes PAHs e fuligem ou apenas fuligem. Os resultados mostram que na região mais fria, perto da entrada de combustível, apenas existem PAH. Seguindo esta região, numa zona de altura intermediária e mais quente, tanto a fuligem como o PAHs coexistem até a o ponto de máxima fração volumétrica integrada de fuligem. O deslocamento no sentido vertical da distribuição de fluorescência é observado com o aumento do comprimento de onda de detecção, o que é consistente com o crescimento do tamanho de PAH e sua progressiva transformação em fuligem. A distribuição de PAH e da fuligem é investigada como função da vazão de combustível. A fração volumétrica de fuligem apresenta uma distribuição clássica, cujo valor se incrementa com a vazão de combustível, enquanto que a temperatura medida diminui.

\section{Palavras-chave}

Incandescência induzida por laser; Fluorescência induzida por plano laser; Absorção/emissão moduladas; Atenuação na linha de visada; Deconvolução. 


\section{Abstract}

Cruz Villanueva, Juan José; Figueira da Silva, Luís Fernando (Advisor); Fuentes Castillo, Andrés (Co-advisor). A experimental study of aromatic precursors and soot distribution for a laminar ethylene coflow diffusion flame. Rio de Janeiro, 2017. 141p. Tese de Doutorado - Departamento de Engenharia Mecânica, Pontifícia Universidade Católica do Rio de Janeiro.

This work presents an experimental study of soot and polycyclic aromatic hydrocarbons (PAH) distribution in axisymmetric ethylene-air non-premixed laminar flames using spectroscopic diagnostic in a co-flow target burner. For this purpose, are applied laser-induced fluorescence and laser-induced incandescence techniques with UV excitation. Spectral detection bands centered at 340, 400, 450, $500,550 \mathrm{~nm}$ are employed to characterize PAH, using the infrared fluorescence spectra displacement phenomenon with the molecular mass increase. The incandescence is captured at $400 \mathrm{~nm}$ and the laser extinction technique is used to calibrate the signal, and, thus to obtain the soot volume fraction at the reaction zone. The soot spontaneous emitted radiation is used to measure the temperature by the two-color pyrometry technique. The comparison between results with prompt (0 ns) and delayed ( $50 \mathrm{~ns}$ ) detection, with respect to the laser pulse, allows to discriminate the regions between soot precursors (PAH) and soot. The results show that, in the colder region, near the fuel inlet, PAH exist only. Following this region, in an intermediate warmer zone, both soot and PAH appear to coexist until the point of maximum integral soot volumetric fraction. A vertical displacement of the fluorescence distribution with increasing detection wavelength is observed, which is consistent with PAH size growth and with its progressive transformation into soot. PAH and soot distribution are investigated as a function of the fuel flow rate. The soot volumetric fraction exhibits a classical distribution, whose value increases with the fuel flow rate, whereas the measured temperature decreases, exhibiting a singular behavior in the region where the soot is formed.

\section{Keywords}

Laser induced incandescence; Planar laser induced fluorescence; Modulated Absorption/Emission; Line of sight attenuation, Deconvolution. 


\section{Sumário}

1 Introdução 23

1.1. Exposição do problema 23

1.2. Objetivos 28

1.3. Organização do manuscrito 28

2. Revisão Bibliográfica: Formação de hidrocarbonetos aromáticos policíclicos e fuligem em chamas 30

2.1. Estudos em chamas laminares 30

2.2. Estudos em chamas turbulentas 34

2.3. Conclusões parciais 37

3. Embasamento das técnicas de medição 40

3.1. Técnicas baseadas na espectroscopia de emissão da chama 40

3.1.1. Quimiluminescência de radicais químicos 40

3.1.2. Luminosidade natural da fuligem 43

3.1.3. Termometria de duas cores 44

3.1.3.1. Fundamentos teóricos 44

3.1.3.2. Implementação utilizada 45

3.2. Extinção laser (LE) 49

3.3. Técnica de incandescência induzida por laser (LII) 53

3.3.1. Processos físico-químicos associados à incandescência 54

3.3.2. Relação do sinal de incandescência $S_{L I I}$ com a fração volumétrica de fuligem $f_{v} \quad 58$

3.3.3. Excitação do sinal de incandescência $S_{L I I}$ e seu decaimento temporal $\quad 60$

3.3.4. Emissão da incandescência das partículas de fuligem 62

3.4. Técnica de fluorescência induzida por plano laser (PLIF) 63

3.4.1. Fundamentos do fenômeno de fluorescência 64 
3.4.2. Fluorescência induzida por laser de hidrocarbonetos aromáticos policíclicos (LIF-PAH)

3.5. Detecção simultânea dos sinais de incandescência e fluorescência induzida por laser

4. Materiais e métodos experimentais

4.1. Queimador empregado para o estudo da formação da fuligem

4.2. Medidores de vazão mássica

4.3. Montagem experimental para as técnicas de espectroscopias por emissão

4.3.1. Termometria de duas cores 73

4.3.2. Quimiluminescência 74

4.4. Montagem experimental das técnicas excitadas por laser $\quad 75$

4.4.1. Extinção de luz 75

4.4.2. Excitação da incandescência e da fluorescência induzidas por laser

4.4.2.1. Caracterização da distribuição da energia do plano laser por espalhamento Rayleigh

4.4.2.2. Caracterização da energia de excitação da técnica LII fluência

80

4.4.3. Calibração dos resultados de incandescência induzida por laser

4.4.4. Detecção dos sinais espontâneos ou induzidos por laser

4.4.5. Detecção espectral dos PAHs e fuligem 84

4.4.5.1. Possíveis limitações da detecção espectral 85

5. Análise e Discussão dos Resultados

5.1. Condições de operação estudadas

5.2. Estudo da zona reativa mediante a emissão espontânea da chama 
5.2.1. Processo de deconvolução das imagens integrais da câmara

5.2.2. Estudo da emissão espectral da chama 90

5.3. Distribuição de temperatura da fuligem nas chamas 94

5.4. Distribuição de fuligem e de hidrocarbonetos aromáticos policíclicos aromáticos $\quad 97$

5.4.1. Campos de sinais imediatos "prompt" e com atraso "delay" 97

5.4.2. Validação das medidas da fração volumétrica da fuligem 101

5.4.3. Comparação dos sinais "delay" e "fluorescência" 103

5.4.4. Comparação dos sinais "delay" e "fluorescência", sem normalização

5.4.5. Influência da vazão de combustível sobre a formação de fuligem e PAH

5.4.6. Análise das propriedades integrais

6. Conclusões e perspectivas

6.1. Conclusões e contribuições 113

6.2.Sugestões para trabalhos futuros $\quad 115$

$\begin{array}{ll}\text { 7. Referências bibliográficas } & 116\end{array}$

$\begin{array}{ll}\text { Apêndice A } & 130\end{array}$

A.1. Determinação das incertezas de medição 130

A.2. Incerteza da temperatura da fuligem medida pela técnica de termometria de duas cores

A.3. Incerteza das medidas de fração volumétrica de fuligem 132

A.4. Incerteza do número de Reynolds do jato de combustível 134

Apêndice B 136

B.1. Queimador de chama plana laminar McKenna 136

B.2. Formação de hidrocarbonetos aromáticos policíclicos e fuligem em chamas laminares pré-misturadas de etileno e ar 
B.3. Variação do sinal de fluorescência e incandescência devido à detecção em diferentes faixas espectrais

B.4. Comparação temporal dos sinais medidos em diferentes faixas espectrais 


\section{Lista de figuras}

Figura 1 - Imagem da (a) estrutura de um aglomerado de fuligem obtida por TEM e (b) etapas de formação da fuligem, adaptado de [1]

Figura 2 - Mecanismo HACA de formação de pireno (A4) a partir de benzeno (A1) [17]

Figura 3 - Espectros normalizados de uma chama de GLP e ar para mistura (a) pobre $\phi=0,81$ e (b) rica $\phi=2,02$, Adaptado de [48]

Figura 4 - Potência emissiva espectral de corpo negro a diferentes temperaturas

Figura 5 - Emissão integrada de uma chama axisimétrica, (a) perfil horizontal e (b) discretização da seção transversal da chama, adaptado de [65]

Figura 6 - Extinção da luz devido a uma nuvem de partículas, adaptado de [62]

Figura 7 - Processos de transferência de energia num aglomerado de partículas de fuligem, adaptado de [85]

Figura 8 - Perfil temporal do sinal de incandescência $S_{L I I}$, temperatura da fuligem (símbolos) obtidos com um pulso laser (linha tracejada) de $532 \mathrm{~nm}$ e fluência de $0,061 \mathrm{~J} / \mathrm{cm}^{2}$ [102]

Figura 9 - Efeitos da fluência do feixe laser: (a) perfil transversal do laser para dois tipos de feixe laser, adaptado de [104] e (b) curva da fluência, $S_{L I I}$, para um laser de perfil gaussiano, adaptado de [105]

Figura 10 - Decaimento temporal da sinal $S_{L I I}$ numa chama laminar de eteno-ar. Diâmetro médio de partículas de $64 \mathrm{e}$ $160 \mathrm{~nm}$ para alturas de 10 e $30 \mathrm{~mm}$ respectivamente [106] 
Figura 11 - Bandas de emissão de Swan (LIF-C2) e curvas de radiação de corpo negro para $4000 K$ e $5000 K$, temperaturas típicas de emissão de LII, numa chama difusiva de etileno. O pico de emissão em $516,5 \mathrm{~nm}$ foi escalado para 0,5 da transmissividade do sistema de detecção fitro-câmara CCD utilizado por [107]

Figura 12 - Diagrama de níveis de energia num processo de fluorescência induzida, adaptado [110]

Figura 13 - (a) Espectros de fluorescência de vários PAHs na fase vapor adaptado de [31] e (b) posição das bandas com máxima fluorescência conforme se incrementa o peso molecular, adaptado de [114]

Figura 14 - Esquema da (a) evolução temporal da fluorescência e incandescência detectadas na faixa UV-Visível e (b) janelas temporais de detecção das câmaras, adaptado de [118]

Figura 15 - Chama não pré-misturadas de etileno e de ar, queimador Gülder e corte transversal do queimador

Figura 16 - (a) Sistema de captura empregado na técnica de termometria por duas cores e (b) curva de eficiência quântica espectral do sensor CCD, adaptado de [128]

Figura 17 - (a) Sistema de captura da quimiluminescência de radicais e (b) curva de eficiência quântica espectral $Q E_{I R O}$ e sensibilidade radiante do fotocátodo $[\mathrm{mA} / W]$ da ICCD, adaptado de [129]

Figura 18 - Montagem experimental da técnica de extinção da luz 76 Figura 19 - Sistema para a excitação e detecção simultânea da fluorescência e incandescência induzida por laser

Figura 20 - Distribuição da energia normalizada do plano laser, $I_{\text {normr }}(z)$, e seu perfil longitudinal em $r=0 \mathrm{~mm}$, obtida por espalhamento Rayleigth

Figura 21 - Curva de fluência da fuligem medida para o caso B 
Figura 22 - Esquema da calibração dos resultados de incandescência induzida por laser $\lambda_{\text {exc }}=266 \mathrm{~nm}$ pela extinção de luz $\lambda_{\text {det }}=532 \mathrm{~nm}$

Figura 23 - Campos normalizados da emissão, a $433 \pm 10 \mathrm{~nm}$, do Caso B (a) integral e deconvoluído e (b) Perfis radiais a diferentes alturas

Figura 24 - Emissão normalizada, Caso B, observada a diferentes bandas espectrais (a) $310 \mathrm{~nm}\left(O H^{*}\right)$, (b) $433 \mathrm{~nm}\left(\mathrm{CH}^{*}\right)$, (c) $530 \mathrm{~nm}$ (fuligem) e (d) $900 \mathrm{~nm}$ (fuligem)

Figura 25 - Perfis radiais da emissão normalizada, caso B, para as alturas adimensionais (a) $z / h_{f}=0,4$, (b) $z / h_{f}=0,6$ e (c) $z / h_{f}=0,8$

Figura 26 - Perfil radial de temperatura, $z / h_{f}=0,46$, comparados com os resultados de Gülder et al. por CARS [124], de Snelling et al. por emissão de comprimentos de onda múltipla [68] e Escudero et al. e por termometria duas cores [59]

Figura 27 - Campos e iso-linhas de temperatura, (a) Caso A, (b) Caso B e (c) Caso C

Figura 28 - Perfis radiais de temperatura a três alturas adimensionais, $z / h_{f}=0,4$ (linha continua), 0,6 (linha tracejada) e 0,8 (linha traço-ponto), (a) Caso A, (b) Caso B e (a) Caso C

Figura 29 - Perfis radiais de temperatura da fuligem para (a) $z / h_{f}=0,6$ e (b) $z / h_{f}=0,8$ e diferentes vazões de etileno

Figura 30 - Campos de sinais $S_{0}^{\lambda_{\text {det }}}$ (esquerda) e $S_{50}^{\lambda_{\text {det }}}$ (direita) obtidos nos comprimentos de onda de $\lambda_{\text {det }}=340 \mathrm{~nm}, 400 \mathrm{~nm}$, $450 \mathrm{~nm}, 500 \mathrm{~nm}$ e $550 \mathrm{~nm}$. Caso B

Figura 31 - Campos de $S_{0}^{\lambda_{\text {det }}}$ na base da chama do caso B, na região $z<-13\left[(r / 4)^{2}-1\right]$, para todas as bandas espectrais de detecção $\lambda_{\text {det }}$ empregadas 
Figura 32 - Campo de $S_{0}^{\lambda_{\text {det }}}$ na base da chama do caso B, mostrado por iso-linhas de $70 \%$ do máximo de intensidade normalizado, para diferentes $\lambda_{\text {det }}$

Figura 33 - Perfil radial de fração volumétrica de fuligem em $f_{v}$, $z / h_{f}=0,65$. Comparação entre os resultados do presente estudo, e os de Hadef et at. [24]

Figura 34 - Fração volumétrica de fuligem do Caso $B$, resultados simetrizados: (a) campo de $f_{v}(r, z)$ e (b) perfis radiais para as às alturas adimensionais, $z / h_{f}=0,2,0,4,0,6,0,8$

Figura 35 - Comparação dos resultados de $S_{(0 n s-50 n s)}^{\lambda_{\text {det }}}$ e $S_{50 n s}^{\lambda_{\text {det }}}$ em diferentes alturas adimensionais $z / h_{f}$ (a) 0,2, (b) 0,4, (c) 0,6 e (d) 0,8 , Caso B

Figura 36 - Intensidades de fluorescência e fração volumétrica de fuligem ao longo da linha central, $r=0$, (a) $S_{0 n s}^{\lambda_{d e t}}$ e (b) $S_{0 n s}^{400}$, Caso B

Figura 37 - Comparação dos resultados de $S_{(0 n s-50 n s)}^{\lambda_{\text {det }}}$ em diferentes alturas (a) $z=6,3 \mathrm{~mm}$, (b) $z=12,6 \mathrm{~mm}$, (c) $z=19 \mathrm{~mm}$ e (d) $z=25,3 \mathrm{~mm}$, Caso B

Figura 38 - Intensidades de fluorescência ao longo da linha central, $r=0$, (a) $S_{(0 n s-50 n s)}^{\lambda_{d e t}}$, Caso B

Figura 39 - Influencia do aumento da vazão de combustível sobre os resultados "promt-delay" e "delay" a duas diferentes alturas adimensionais $z / h_{f}$ (a) 0,2 , (b) 0,4

Figura 40 - Fração volumétrica de fuligem e temperatura ao longo da linha de corrente $r=0$, para as vazões de etileno $V_{f}$ de (a) $80 \mathrm{sccm}$, (b) $100 \mathrm{sccm}$ e (c) $140 \mathrm{sccm}$

Figura 41 - Influência da vazão de etileno sobre a fração volumétrica integral da fuligem (a) função de $z$ e (b) função de $z / h_{f}, \lambda_{\text {det }}=400 \mathrm{~nm}$ 
Figura 42 - Resultados de estimação da incerteza experimental para o Caso G. Campos de (a) Temperatura, (b) incerteza absoluta $\delta T$ e (c) incerteza relativa $\delta T / T$

Figura 43 - Chama laminar de etileno e ar gerada num queimador McKenna e estabilizada por uma placa circular de aço [120]

Figura 44 - Sinal detectado imediatamente após excitação laser para diferentes faixas de detecção espectral

Figura 45 - Campos médios dos sinais detectados sem atraso para (a) $\lambda_{\text {det }}=340 \mathrm{~nm}$ e (b) $\lambda_{\text {det }}=400 \mathrm{~nm}$

Figura 46 - Evolução longitudinal do sinal normalizada em $x=0$, para diferentes atrasos, nas bandas de $340 \mathrm{~nm}$ e $400 \mathrm{~nm}$

Figura 47 - Evolução temporal dos sinais normalizados com o tempo de atraso da detecção para diferentes alturas a partir da superfície do queimador 


\section{Lista de Tabelas}

Tabela 1 - Resumo dos estudos apresentados para chamas laminares

Tabela 2 - Características das técnicas de diagnostico empregadas

Tabela 3 - Modelos de medidores de vazão ALICAT empregados neste estudo

Tabela 4 - Parâmetros utilizados na técnica de pirometria duas cores

Tabela 5 - Parâmetros utilizados na medição da

Tabela 6 - Filtros passa-banda (Edmund Optics) utilizados na medição simultânea de LIF-PAHs e LII

Tabela 7 - Condição de operação dos casos estudados. $V_{a r}=$ $60 \pm 0,68 \mathrm{slpm}$

Tabela 8 - Valores de incerteza das variáveis empregadas no cálculo de $f_{v}$

Tabela 9 - Valores de incerteza das variáveis empregadas no cálculo do número de Reynolds do jato de etileno 


\section{Nomenclatura}

\section{Caracteres Latinos}

$a$

$: \pi d_{p} / \lambda_{\text {exc }}$.

$b(z) \quad:$ Fator de escala para o sinal atrasado $S_{50 \mathrm{~ns}}^{\lambda_{\text {det }}}$.

$E(m) \quad$ : Função de absorção do índice de refração, $E(m)=0,2395$.

f : Distância focal da lente.

h : Variação de Entalpia.

$I_{0} \quad$ : Intensidade do feixe de extinção incidente.

I : Intensidade do feixe de extinção passante.

$l \quad$ : Coordenada longitudinal, ao longo do caminho óptico que passa por $y$.

$L \quad$ : Caminho óptico total da chama atravessada pelo laser de extinção na coordenada $y$.

$m \quad$ : Índice de refração complexo, $m=1,7316-i 0,6$.

$N\left(d_{p}\right) \quad$ : Função de distribuição do diâmetro das partículas.

$N \quad$ : Número de anéis da seção transversal da chama.

$r \quad$ : Coordenada radial.

$R_{f} \quad$ : Raio da chama numa determinada altura.

$t \quad:$ Tempo.

T : Temperatura.

y : Coordenada de posicionamento do caminho óptico.

$W(t) \quad$ : Função de amostragem.

\section{Caracteres gregos}

$\bar{\beta} \quad$ : Sinal de fluorescência ou incandescência radialmente integrado.

$v \quad$ : Frequência de radiação.

$\phi \quad$ : Função trabalho na emissão termoiônica.

$\tau \quad$ : Transmitância.

$\Omega \quad$ : Ângulo solido. 


\section{Subscritos e sobrescritos}

$C_{s} \quad:$ Calor específico da fuligem.

$C_{n} \quad:$ Número de partículas por unidade de volume.

$C_{P} \quad:$ Calor especifico a pressão constante.

$C_{V} \quad:$ Calor especifico a volume constante.

$d_{P} \quad$ : Diâmetro da partícula primaria de fuligem.

$\dot{E}_{a b s} \quad$ : Taxa de aquecimento por absorção.

$\dot{E}_{\text {rad }} \quad$ : Taxa de resfriamento por radiação.

$\dot{E}_{\text {cond }} \quad$ : Taxa de resfriamento por condução.

$\dot{E}_{\text {Sub }} \quad$ : Taxa de resfriamento por sublimação.

$\dot{E}_{o x} \quad$ : Taxa de aquecimento por sublimação.

$\dot{E}_{\text {rec }} \quad$ : Taxa de aquecimento por recozimento.

$\dot{E}_{t e r} \quad$ : Taxa de resfriamento por emissão termoiônica.

$f_{v} \quad$ : Fração volumétrica de fuligem.

$\bar{f}_{v} \quad$ : Fração volumétrica média de fuligem.

$F_{v} \quad: F_{v}=\int_{0}^{L} f_{v} d r$ Fração volumétrica integral ao longo do caminho óptico.

$f_{v}(t) \quad$ : Fração volumétrica instantânea de fuligem.

$\bar{f}_{v}(t) \quad:$ Fração volumétrica média de fuligem.

$f_{v, \max }(t) \quad$ : Máximo da fração volumétrica instantânea de fuligem.

$G_{e}(t) \quad:$ : Irradiação temporal do laser.

$h_{f} \quad$ : Altura da chama.

$h_{P} \quad$ : Posição vertical do mínimo local de $S_{0}^{\lambda_{\text {det }}}(0, z)$.

$\Delta H_{V} \quad:$ : Entalpia de formação de um aglomerado de partículas sublimado.

$\Delta H_{O X} \quad$ : Entalpia da reação $C+1 / 2 O_{2} \rightarrow C O$.

$\Delta H_{a n n} \quad$ : Entalpia de recozimento.

$I_{\lambda} \quad:$ Intensidade de radiação espectral.

$I_{\lambda}^{e} \quad$ : Intensidade de radiação espectral acumulada.

$I_{\lambda}^{b b} \quad$ : Intensidade de radiação de corpo negro.

$J_{\lambda} \quad$ : Emissão monocromática da chama ou da fuligem. 
$J_{\lambda}^{e}(y) \quad$ : Emissão espectral acumulada ao longo do caminho óptico, na coordenada y.

$K_{e} \quad:$ Coeficiente adimensional de extinção, $K_{e}=6 \pi E(m)=4,51$.

$m_{c} \quad:$ massa do elétron.

$\dot{m}_{f} \quad$ : Vazão mássica de combustível.

$N_{d} \quad:$ Fator de consideração de defeitos.

$P_{0} \quad$ : Pressão do ambiente.

$P_{i}(y) \quad$ : Perfil de intensidade acumulada.

$p_{V} \quad$ : Pressão parcial dos aglomerados de carbono.

$Q_{e x} \quad:$ Eficiência de extinção.

Q : Eficiência de absorção.

$Q_{\text {scat }} \quad$ : Eficiência de espalhamento.

$Q E_{C C D} \quad$ : Eficiência quântica do sensor da câmara CCD.

$Q E_{I C C D} \quad$ : Eficiência quântica do sensor do intensificador IRO.

$S_{d t}^{\lambda_{d e t}} \quad:$ Sinal emitido (LII, LIF ou luminosidade) na faixa espectral $\lambda_{\text {det }}$.

$S_{\text {detec }}^{\lambda_{\text {det }}} \quad:$ Sinal detectado pela câmara na faixa espectral $\lambda_{\text {det }}$.

$S_{(0 n s-50 n s)}^{\lambda_{\text {det }}} \quad:$ Sinal da fluorescência de PAH.

$S_{L I I} \quad:$ Sinal de incandescência induzida por laser.

$\overline{S_{L I I}} \quad:$ Sinal de incandescência médio ao longo do caminho óptico.

$S_{P A H} \quad:$ Sinal de fluorescência induzida por laser.

$T_{a d} \quad$ : Temperatura da chama adiabática $\left(2369 \mathrm{~K}\right.$ para o $\left.\mathrm{C}_{2} \mathrm{H}_{4}\right)$.

$T_{f} \quad:$ Temperatura das moléculas depois do impacto com a superfície.

$T_{0} \quad:$ Temperatura do ambiente.

$u_{f} \quad:$ Velocidade média do escoamento de combustível.

$\dot{V}_{f} \quad$ : Vazão de combustível.

$V_{m v} \quad$ : Volume de medição de LII ou PLIF.

$W_{a} \quad$ : Massa molecular do ar.

$W_{V} \quad:$ Massa molecular média de um aglomerado de partículas sublimado.

$W_{1} \quad$ : A massa molecular de um átomo de carbono.

$Z_{\text {surf }} \quad$ : Taxa de colisão molar do gás.

$\alpha_{M} \quad$ : Coeficiente de acomodação mássica dos aglomerados vaporizados. 
$\alpha_{T}$

$\varepsilon_{\lambda}$

$k_{a b s}$

$k_{B}$

$k_{e x}$

$k_{o x}$

$k_{\text {ann }}$

$\lambda_{\text {exc }}$

$\lambda_{\text {ext }}$

$\lambda_{\text {det }}$

$\rho_{s}$

$\rho_{f}$

$\sigma_{a b s}$

$\tau_{\text {res }}$

$\tau_{F}$

$\Phi_{\text {glob }}$

: Coeficiente de acomodação térmica.

: Emissividade espectral da chama ou da fuligem.

: Coeficiente de absorção.

: Constante de Boltzmann.

: Coeficiente de extinção.

: Taxa da reação $2 \mathrm{CO}+\mathrm{O}_{2} \rightarrow 2 \mathrm{CO}$.

: Constante do modelo de recozimento.

: Comprimento de onda de excitação laser.

: Comprimento de onda do laser de extinção.

: Comprimento de onda de detecção.

: Densidade da partícula de fuligem.

: Densidade do combustível.

: Seção transversal de absorção.

: Tempo de residência da chama $\left(h_{f} / u_{f}\right)$.

: Tempo de vida da fluorescência.

: Riqueza global.

\section{Constantes}

c

$C_{1}$

$C_{2}$

$C_{c a l}$

$h$

$N_{a}$

$R$

\section{Siglas}

BB : Bluff-body.

CCD

CMD

HACA : Mecanismo abstração de hidrogênio - adição de acetileno ICCD : Intensified charge-coupled device.

: Velocidade da luz.

: Primeira constante da equação de Planck.

: Segunda constante da equação de Planck.

: Constante de calibração.

: Constante de Planck.

: Constante de Avogadro.

: Constante universal dos gases.

\section{: Charge-coupled device.}

: Contagem do diâmetro médio da distribuição log-normal do $d_{p}$. 
IRO : Intensified relay optics.

JP : Jato de precessão.

JC-LIF : Técnica LIF por jato resfriado.

LE : Extinção laser.

LII : Incandescência induzida por laser.

PAH : Hidrocarbonetos aromáticos policíclicos.

PLIF : Fluorescência induzida por plano laser.

PDF : Função densidade de probabilidade.

TEM : Microscópio eletrônico de transmissão.

REMPI : Ressonância comportada por ionização multifóton.

SJ : Jato simples.

TER : Transferência de energia rotacional.

TOF-MS : espectroscopia de massa por tempo de voo. 